\title{
Pueblos indígenas, recursos y gobernanza. Un análisis de la consulta indígena como parte de la Evaluación de Impacto Ambiental del proyecto hidroeléctrico Añihuerraqui, Región de la Araucanía, Chile
}

Indigenous peoples, resources and governance. An analysis of the Indigenous consultation as part of the Environmental Impact Assessment of the Añihuerraqui hydroelectric project, Araucanía Region, Chile

\author{
Historial del artículo \\ Recibido: \\ 24 de marzo de 2020 \\ Revisado \\ 14 de mayo de 2020 \\ Aceptado:
}

25 de mayo de 2020

\author{
Johanna Höhl ${ }^{\mathrm{a}}$ \\ a Heidelberg Center para América Latina. Centro de Excelencia en Investigación y Docencia, Chile. Correo electrónico: \\ j.hoehl@heidelbergcenter.cl
}

Esta investigación cuenta con financiamiento del Proyecto Fondecyt de Iniciación 11180970
Palabras clave

Chile, gobernanza, hidroelectricidad, pueblos indígenas

\section{Keywords}

Chile, governance, hydroelectrocity, indigenous peoples

\begin{abstract}
Resumen
La gobernanza permite aproximarse a las interacciones entre el Estado, el mercado y la sociedad civil de forma holística al incluir en el análisis del desarrollo social grupos no tradicionales. Esta perspectiva teórica considera a los pueblos indígenas como un actor relevante en los procesos de toma de decisión en torno al uso de los recursos naturales. La consulta indígena creada como parte de la institucionalidad ambiental de Chile en el año 2013 fue concebida como espacio de articulación y negociación con el objetivo de empoderar las posturas y demandas indígenas. Sin embargo, las diferentes visiones de la naturaleza que se articulan en el marco de la consulta indígena complejizan el desarrollo de la evaluación de impacto ambiental en territorios indígenas. Asimismo, el mecanismo no considera la heterogeneidad dentro y entre las comunidades indígenas en un mismo territorio, ni cumple con asignarles un mayor control sobre los recursos naturales en sus territorios. Frente a ese escenario cabe preguntarse cómo se valida y legitima la consulta indígena frente a los pueblos indígenas y cuál es su contribución a la redefinición de su rol en los procesos de negociación en torno a proyectos extractivos en sus territorios. Mediante el análisis de la consulta indígena como parte de la Evaluación de Impacto Ambiental del proyecto hidroeléctrico Añihuerraqui en este artículo se evidencia la división de las comunidades indígenas afectadas, así como la limitación de su participación en el proceso de toma de decisión generando incertidumbre entre los actores involucrados.
\end{abstract}




\section{Introducción}

En los últimos años el número de conflictos ambientales en América Latina ha ido en aumento. Con la consolidación del modelo neoliberal desde la década de los años ochenta, la expansión de la minería, las empresas forestales, la agroindustria, pero también del sector energético, ha causado conflictos entre el Estado, el sector privado y las comunidades locales afectadas por los proyectos neoextractivistas asociados (Bebbington, 2012; Peralta, Bebbington, Hollenstein, Nussbaum \& Ramírez, 2015). La ecología política ha evidenciado cómo los diferentes intereses en cuanto al desarrollo económico y también el uso de los recursos naturales se manifiestan en este tipo de disputas (Castillo, Espinoza \& Campos, 2017), y cual es el rol de las distintas relaciones hombre-naturaleza (Swyngedouw, 2004; Swyngedouw, 2011; Swyngedouw \& Copano, 2018), específicamente por parte de los pueblos indígenas (Escobar 2006; Ulloa, 2020) en estos contextos. La mayoría de los Estados latinoamericanos han ratificado el Convenio 169 de la OIT sobre pueblos indígenas y tribales (C169), y a raíz de lo mismo implementaron procesos de consulta frente al desarrollo de proyectos extractivistas (Walter \& Urkidi, 2011). Chile se sumó recién en el año $2008^{1}$, y consecuentemente el Estado chileno ha tenido que considerar los pueblos indígenas en las decisiones que afectan directamente a sus territorios y reconocer así, entre otras, sus formas de vida y prácticas culturales. En esa línea para cumplir con lo requerido en el C169, el Estado chileno creó la llamada consulta indígena que forma parte del Sistema de Evaluación Ambiental (SEIA) desde el año 2013 (Leppe, 2015). La consulta indígena es definida como un proceso participativo en el marco de la evaluación de impacto ambiental (EIA) de un proyecto minero, energético, forestal u otro. Así, este mecanismo apunta a canalizar el choque entre diferentes concepciones de la naturaleza e intereses en torno al uso de los recursos naturales -globales y locales, indígenas y no indígenas- pero también a fomentar el diálogo entre distintos grupos de actores y sus respectivos intereses. Sin embargo, ¿Cómo se valida y legitima la consulta indígena frente a los pueblos indígenas? ¿Cómo contribuye a la redefinición del rol de los pueblos indígenas en los procesos de negociación en torno a proyectos extractivos en sus territorios? Para responder a estas preguntas, el presente artículo busca contribuir a un mayor entendimiento de las complejidades presentes en los procesos de toma de decisión respecto al desarrollo hidroeléctrico a partir del caso de la central de pasada Añihuerraqui en la Región de la Araucanía en el sur de Chile, mediante un análisis desde la gobernanza, concepto que parte de la base de que el Estado no es capaz de resolver problemas ambientales complejos por sí solo (Kooiman, 1999; Rhodes, 1996). La implementación de la consulta indígena permite, por lo tanto, analizar procesos de toma de decisión y las estructuras asociadas, y así identificar los desafíos que presenta la inclusión de un mayor número de actores, y sobre todo de actores con posiciones e intereses divergentes a la dominante.

Así, el presente trabajo complementa investigaciones realizadas desde la ecología política respecto a los conflictos socioambientales en América Latina.

\section{Gobernanza - una aproximación teórica}

Desde mediados de los años noventa la gobernanza ha tenido un auge y el término ha sido utilizado tanto por geógrafos y sociólogos como por cientistas políticos (Bridge \& Perreault, 2009; Pierre \& Peters, 2000). La gobernanza presenta diferentes ramas, la ambiental es una de ella, enfocada en problemas ambientales complejos, tales como la creciente presión sobre el agua, el cambio climático, los cambios de usos de suelos o los impactos ambientales por proyectos extractivos. Al entender el concepto desde una perspectiva prescriptiva, la solución a los problemas ambientales mencionados debe buscarse en conjunto con el Estado, el sector privado y la sociedad civil (Mette Kjaer, 2011; Treib, Bähr \& Falkner, 2007). Este giro desde la teoría en cuanto a quienes deberían participar en la resolución de asuntos complejos está estrechamente vinculado a la disminución de la influencia de los gobiernos centrales a favor de los mercados que se inició con los procesos de privatización y desregularización desde los años 1970 (Harvey, 2007). Esto provoca que las esferas públicas y privadas ya no son vistas como algo separado, sino que interrelacionado.

Con base en esta idea, Rhodes (1996) se refiere a la gobernanza como un concepto que toma en consideración varios actores, que incluye aquellos no vinculados al Estado, que forman parte de procesos de negociación e interrelación en torno a la asignación de recursos. Es justamente esa inclusión de un amplio espectro de actores y de sus interrelaciones en el análisis de procesos de

${ }^{1}$ Ver Indigenous and Tribal Peoples Convention, C169. 
gobernanza que acorde a Pierre \& Peters (2000) contribuye a la popularidad del concepto. El giro cualitativo en la investigación de procesos de toma de decisión, así como la consideración del rol de grupos alternativos de la sociedad en la coordinación económica y política, acompaña esa reorientación del análisis de los problemas sociales (Bridge \& Perreault, 2009). En el caso de la consulta indígena, la perspectiva de la gobernanza no solo amplía las variables de análisis, sino que también facilita la visibilización de las demandas indígenas por un mayor reconocimiento y representación política.

Sin embargo, la gobernanza no solo considera un amplio espectro de actores y sus intereses en el marco de los procesos de toma de decisión, sino que permite también analizar cómo los diferentes actores interactúan con base en leyes, políticas, normas, costumbres y códigos de comportamiento, lo que permite documentar transformaciones en los procesos de toma de decisión (Berkes, 2010; Bridge \& Perreault, 2009), así como entender las prácticas sociales (de Loë \& Patterson, 2017). Por lo tanto, el concepto teórico comprende tanto la interacción entre diferentes actores, así como las reglas y prácticas formales e informales que conforman estas relaciones (Pierre \& Peters, 2000). En consecuencia, la gobernanza como marco analítico permite identificar, por un lado, los procesos y, por el otro, las estructuras que marcan las relaciones entre los grupos indígenas y otros actores, pero también entre diferentes actores pertenecientes a los pueblos indígenas (Von der Porten, de Loë \& Plummer, 2015) que pueden competir por diferentes usos de los recursos naturales o bien tener diferentes intereses (Li, 2010).

En el presente trabajo se afirma que son los legados históricos en torno a la distribución de poder, así como los valores asociados que influyen los procesos y estructuras de decisión y la interacción entre grupos indígenas y otros actores, pero también dentro del mismo grupo (Armitage, 2008). Por lo tanto, el análisis de la consulta indígena creada como un proceso de negociación y mediación en torno al uso de recursos naturales (Donoso, 2014) contribuye a la generación de conocimiento sobre nuevas geografías "de identidad, pertenencia y espacio" (Coombes, Johnson \& Howitt, 2013, p. 694) causadas por actividades mineras, forestales, agroindustriales e hidroeléctricas en territorios indígenas en Chile.

\section{El rol de diferentes socionaturalezas en los procesos de gobernanza}

La transformación de las relaciones hombre-naturaleza a raíz de la expansión de la minería, las empresas forestales, pero también del sector energético en Chile impacta las diferentes relaciones socionaturales. Existen distintas formas de producir naturaleza con base en factores económicos y ecológicos, pero también una gran variedad de prácticas de apropiación y uso de la naturaleza (Swyngedouw, 2011). Por lo tanto, las relaciones socionaturales tienen significados culturales, por lo que pueden ser asociados a diferentes construcciones de identidades (Leff, 2000). Diferentes grupos de personas movilizan la naturaleza para demarcarse y delimitar sus identidades. Así se construyen diversos grupos que entran en conflicto al momento de concebirse mutuamente como in- y outgroup (Lillo \& Nolden, 2003). Sin embargo, la construcción de las identificaciones con los diferentes grupos es dinámica y se realiza mediante procesos interactivos. A lo largo del tiempo y dependiendo de la situación, la pertenencia e identificación con un grupo puede cambiar (Höhl, 2015). Para los pueblos indígenas la naturaleza es una fuente de vida y sagrada; un elemento esencial para la construcción de sus identidades étnicas. Desde su punto de vista lo no humano tiene un valor no traducible a valores económicos, lo que se contradice con la visión económica neoliberal que mercantiliza la naturaleza no solo para su explotación, sino también para poder cuantificar los impactos causados por la actividad humana (Ulloa, 2020).

Por lo tanto, las perspectivas indígenas sobre el desarrollo (económico) en sus territorios desafían las aproximaciones hegemónicas y son movilizadas en disputas por diferentes usos de los recursos en el territorio (Escobar, 2006) que alteran las relaciones históricamente establecidas entre la comunidad y el medio ambiente (Aliste, Folchi \& Núñez, 2018). Es decir, frente a conflictos socioambientales diferentes identidades se asocian a ciertas convicciones y aspiraciones en torno a la naturaleza, que al atribuirles las características "bueno" y "malo" con el fin de imponer una opción sobre la otra para justificar un modelo y uso del recurso frente al otro (Peluso, 2012) causando conflictos. Los ejes centrales de estas disputas son identidades, el medio ambiente y el modelo de desarrollo (Escobar, 2006; Latta \& Wittman, 2010). Es efectivamente el acompañamiento de las relaciones socionaturales por la construcción de identidades étnicas, así como aspiraciones a autodeterminación y autonomía que agudizan los conflictos. La consulta indígena busca hacerse cargo de estas diferencias y ofrecer un espacio para la articulación y negociación de distintas posiciones y visiones que prosperan y se movilizan al momento de presentarse diferentes intereses en torno al control, el uso de recursos naturales, y los impactos asociados en territorios indígenas. Así, la consulta indígena como proceso participativo apunta a la disolución de tensiones mediante la conciliación del poder de los diferentes grupos. 


\section{La consulta indígena como proceso de gobernanza}

La consulta indígena se enmarca en la legislación ambiental e indígena chilena actual. Ambas tienen su origen en los años noventa con el retorno a la democracia cuando tanto los pueblos indígenas como los movimientos ambientales comienzan a articularse fuertemente. Los pueblos indígenas no solo se posicionan como grupos con percepciones e intereses diferentes demarcándose como "otros", usando factores como su relación con el medio ambiente, sino que también buscan visibilizar sus demandas hacia una mayor autodeterminación y autonomía en sus territorios (Kaltmeier, 2004). Es decir, los grupos indígenas buscan empoderarse, para así aumentar su influencia en los procesos de toma de decisión en torno a la aplicación del modelo de desarrollo hegemónico en sus territorios, y el uso de los recursos naturales. Frente a estos avances, el Estado responde mediante el así llamado "nuevo trato de los pueblos indígenas" que se traduce en la proclamación de la Ley Indígena y la creación de la Corporación Nacional de Desarrollo Indígena (CONADI) en el año 1993 (Levil, 2006).

Asimismo, el Estado chileno responde a las demandas de los movimientos ambientales por una mayor protección del medio ambiente frente a la explotación de recursos naturales y el desarrollo económico (Rojas, Sabatini \& Sepúlveda, 2003). En el año 1994, el gobierno aprueba la Ley sobre Bases Generales de Medio Ambiente, que establece la creación de la Comisión Nacional de Medio Ambiente (CONAMA) (Rivera, 2011). En este mismo contexto es creado el SEIA que comienza a operar de forma obligatoria en abril de 1997 (Rojas et al., 2003). Sin embargo, tanto la CONADI como la CONAMA, pero también el SEIA, han sido subordinados a los intereses hegemónicos del sistema neoliberal, es decir, al crecimiento económico por sobre la protección del medio ambiente y los derechos indígenas (Höhl, 2015; Rivera, 2011).

Sin embargo, la ratificación del C169 en el año 2008 y la transformación de la legislación ambiental, así como la creación del Ministerio de Medio Ambiente en el año 2010 presentan un giro en la importancia del medio ambiente y los pueblos indígenas en el panorama institucional chileno. En este marco la implementación de la consulta indígena apunta a la influencia de los pueblos indígenas en el control y uso de los recursos naturales en sus territorios (Leppe, 2015). Por lo tanto, la consulta reúne intereses ambientales e indígenas, apuntando y contrarrestrando a una desfragmentación de la legislación ambiental e indígena. El objetivo principal del mecanismo consiste en la mediación entre diferentes entidades involucradas en proyectos con impacto ambiental en territorios indígenas permitiéndoles articular su posición y solicitar medidas de mitigación, adaptación y reparación, con la idea de empoderar a la población indígena y hacerla partícipe en la toma de decisiones. Así busca fomentar la participación inclusiva de los pueblos indígenas en el proceso de EIA, diferenciándola de la participación ciudadana (Leppe, 2015) con el fin de formalizar un trato diferente en base a una comprensión histórica, política, social, cultural, de territorio y desarrollo distinta.

\section{El ejercicio de la consulta indígena}

La legislación ambiental establece el marco legal para la consulta indígena como parte del proceso de EIA ${ }^{2}$. Los diversos pasos que comprende la consulta indígena se detallan a continuación, ya que tienen implicancias para el análisis de este mecanismo de participación en los procesos de toma de decisión desde una mirada analítica de la gobernanza ${ }^{3}$. El primer paso consiste en la identificación de las comunidades y personas indígenas afectadas por el proyecto ingresado al proceso de EIA por parte de la CONADI con base en los registros que mantiene el organismo respecto a la Ley Indígena. Solo en el caso de que se identifique población indígena debidamente registrada se da curso al proceso de consulta indígena por parte del Servicio de Evaluación Ambiental (SEA) quien está a cargo del SEIA. Es importante recalcar que aquella población que se declara perteneciente a un pueblo indígena, pero no ha hecho los trámites correspondientes frente a la CONADI, no puede participar de la consulta.

Una vez identificados los grupos indígenas afectados, representantes del SEA organizan reuniones con ellos para presentar el proyecto. La CONADI no participa del proceso de consulta como tal (Figura 1). El rol del organismo estatal consiste en asesorar tanto a los grupos indígenas afectados respecto al proceso y sus derechos, como al SEA en torno a cómo comunicar de forma entendible los detalles del proyecto, en el caso de que uno o ambos soliciten el apoyo. Una vez que se hayan reunido e iniciado el proceso, las comunidades indígenas y él SEA acuerdan la metodología de la consulta indígena. Aunque la metodología sigue ciertas reglas, los procesos

\footnotetext{
${ }^{2}$ Ver Ley sobre Bases Generales del Medio Ambiente.)

${ }^{3}$ Los procedimientos y reglas de la consulta indígena como parte de la evaluación de impacto ambiental son fijadas en el decreto 40 que aprueba el reglamento del sistema de evaluación de impacto ambiental en los artículos 85 y 86.
} 


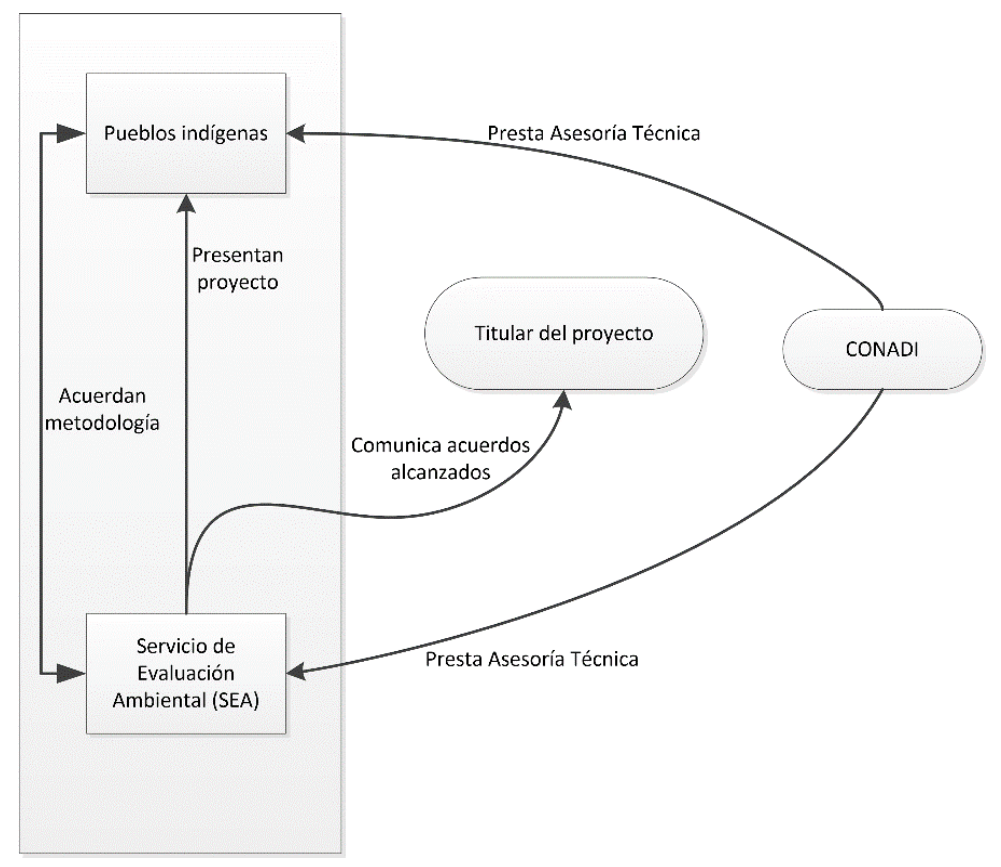

Figura 1. Esquema de la consulta indígena. Fuente: Elaboración propia basada en Barría (2019).

de consulta pueden diferir entre comunidades y personas indígenas afectadas por un mismo proyecto. Asimismo, la consulta indígena varía según el contexto y el proyecto en el cual se desarrolla. Uno de los aspectos que se define en la metodología corresponde a la participación del titular del proyecto, que puede ser el sector privado o el Estado (Barría, 2019). En el caso de que se rechace su participación, el SEA cumple el rol de mediador, quien comunica las solicitudes de los pueblos indígenas, así como los acuerdos alcanzados al titular del proyecto, para que pueda responder a las mismas y puedan ser incorporadas a la Resolución de Calificación Ambiental (RCA).

\section{Metodología}

Este trabajo se enmarca en el análisis de procesos de gobernanza en diferentes contextos que involucran diversos actores (Armitage, 2008) mediante el estudio de un caso concreto. Esto permite responder a preguntas de "por qué" y “cómo", y así entender acontecimientos contemporáneos (Yin, 2009). Concretamente, el estudio del caso de la consulta indígena como parte de la EIA de la central hidroeléctrica de pasada Añihuerraqui, se basa en una exhausta revisión de los documentos disponibles en el SEIA y que corresponden al proceso de EIA, así como de las consultas indígenas que se realizaron en el marco del proceso de calificación ambiental del proyecto. Asimismo, se revisó la RCA. Artículos en diarios referentes al caso, así como trabajos académicos e informes realizados por parte de actores involucrados en los procesos de EIA y de consulta indígena complementaron el análisis de los documentos oficiales. Una visita etnográfica a terreno permitió validar información obtenida mediante los documentos in situ, y así contribuir a la triangulación de los datos de diferentes fuentes. El análisis de los datos se efectuó de forma cualitativa mediante la codificación de los documentos. Esto permitió identificar la complejidad de las relaciones entre y dentro de los actores en una escala local, así como las constelaciones de los actores y su grado de participación en los respectivos procesos (EIA y consulta indígena) con base en el estudio del caso de la hidroeléctrica de pasada Añihuerraqui.

\section{El caso de estudio: el proyecto hidroeléctrico de pasada Añihuerraqui, Región de la Araucanía, Chile}

El proyecto hidroeléctrico de pasada Añihuerraqui fue iniciado por la empresa GTD Negocios S.A., representante en Chile de la compañía española Enhol, y se ubica en el sector precordillerano en los alrededores de Curarrehue (Figura 2), zona con alta presencia mapuche-pehuenche en la Región de la Araucanía en el sur de Chile. Actualmente, 


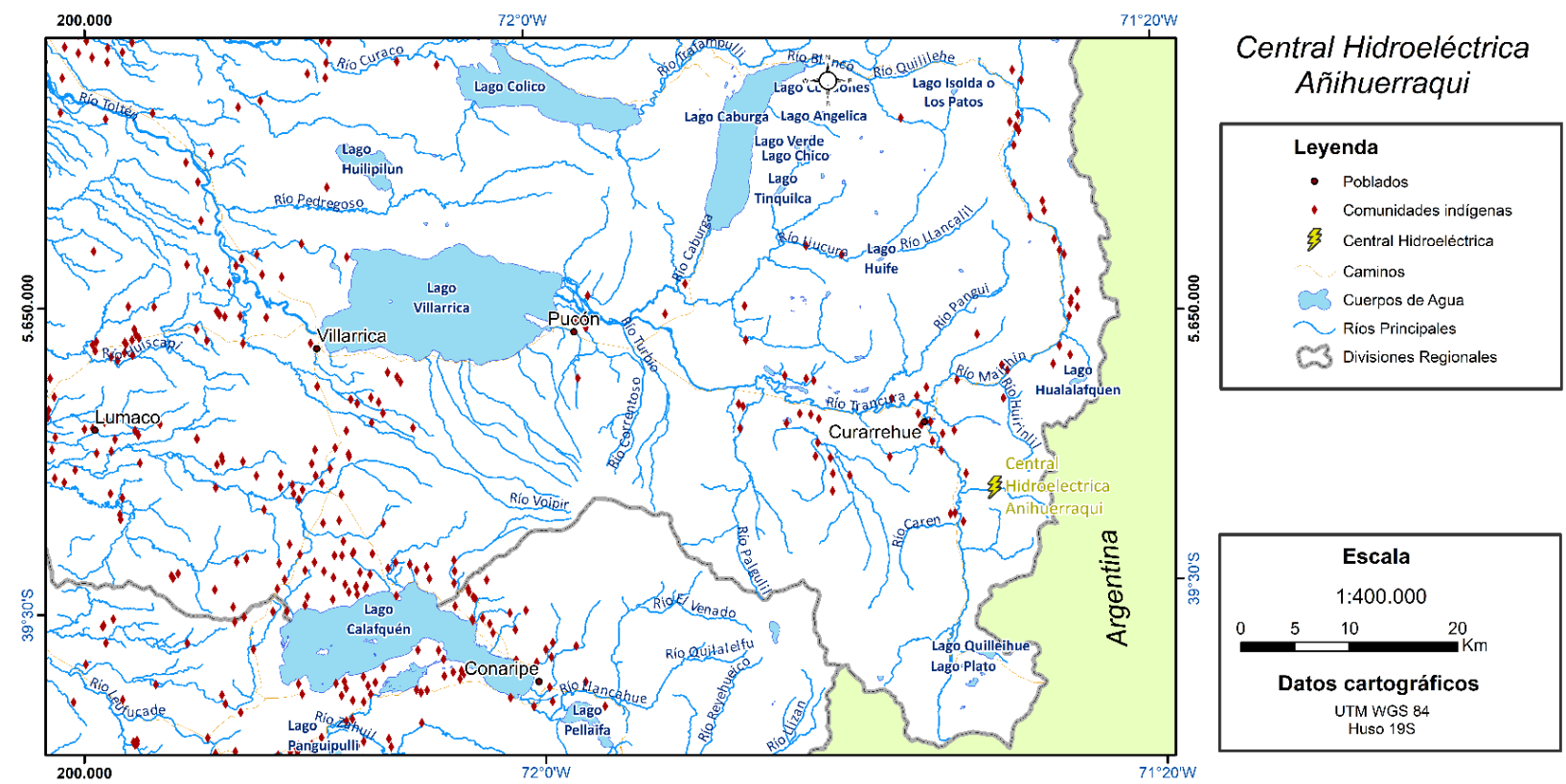

Figura 2. Ubicación proyecto Añihuerraquí. Fuente: Elaboración propia (2019).

el proyecto es ejecutado por Taguavento, empresa ligada a la familia Matte.

El propósito de la inversión de 22 millones de dólares consiste en la instalación de una bocatoma 2,5 km arriba de la unión de los ríos Añihuerraquí y Trankura para luego entubar el agua en 2,4 km, para aprovechar la pendiente de la cordillera junto con el caudal para la producción de $9 \mathrm{MW}$ para ser inyectados al Sistema de Interconexión Central (SIC) (Gestión Ambiental Consultores (GAC), 2012). El proyecto inicia el proceso de EIA en el año 2012. Por enmarcarse en territorio indígena el proyecto debe incluir -como primer proceso- la consulta indígena, que se lleva a cabo en los años 2013 y 2014, aprobándose mediante la RCA en el año 2015. La votación sobre el proyecto se realiza a nivel regional y resulta con siete votos a favor por parte de los Seremis de Transporte, Salud, Obras Públicas, Energía, Economía y Medio Ambiente, así como del SEA; y cuatro votos en contra, por parte de los Seremis de Desarrollo Social, Vivienda y Agricultura, así como por parte de la gobernación (Garrido, 2015).

A consecuencia de la aprobación del proyecto un grupo indígena afectado presenta una querella frente a los tribunales para evitar el inicio de las obras reclamando que el proyecto afecta sitios sagrados, así como la recolección de plantas medicinales (CONADI, 2 de enero de 2013). Al fallar la Corte de Apelaciones de Temuco a favor de la construcción de la hidroeléctrica llevan la demanda hasta la Corte Suprema. Sin embargo, a nivel nacional el recurso es rechazado, reafirmando el fallo de la Corte de Apelaciones dando "luz verde" a la construcción de la Central (Martínez, 2016). Sin embargo, no se trata de un recurso de protección presentado por las comunidades indígenas afectadas en su globalidad. Por el contrario, solo un grupo se querella en contra del proyecto. Esto evidencia la heterogeneidad de las posiciones de las comunidades indígenas afectadas frente al desarrollo económico y los proyectos asociados.

\section{Resultados}

El trabajo demuestra por un lado que la consulta indígena divide a las comunidades, y que por el otro no permite su real participación en el proceso de toma de decisión asociado creando incertidumbre sobre la utilidad de la participación no sólo entre los grupos índigenas, sino que también por parte del titular del proyecto. A continuación, se presentan los principales resultados que consisten, por un lado, en las implicancias de las posiciones heterogéneas frente al proyecto hidroeléctrico Añihuerraquí, y por el otro, en los desafíos que presenta esa heterogeneidad entre 
otros factores como negociaciones previas y asimetrías de información para el proceso de consulta indígena.

\section{Las posturas heterogéneas indígenas}

Mientras que las comunidades Juanita Curipichun de Caren y Camilo Coñoequir deciden negociar sobre medidas de mitigación, adaptación y reparación con el titular del proyecto, previo al proceso de consulta indígena, las comunidades Camilo Coñoequir Lloftunecul y Folilco Juan Curipichun rechazan las propuestas de la empresa y cualquier interacción fuera de la consulta. Desde el punto de vista de los opositores dentro del pueblo indígena las negociaciones previas en la zona con el objetivo de llegar a potenciales acuerdos corresponden a procesos no regulados (GAC, 2012; SEA, 2018). Esto se confirma mediante la posición de defensores del proyecto frente a la consulta indígena: „no requerimos concretar la participación en la Consulta Indígena ya que creemos que puede ser contraproducente, ya que los socios de la comunidad han manifestado (...) no querer participar de este proceso dado que ya contamos con los Acuerdos con la empresa" (SEA, 2018). Sin embargo, las consecuencias de la negociación previa van más allá del rechazo de la consulta indígena por un grupo, provocando la demarcación de otros miembros de una misma comunidad, quienes rechazan el proyecto y no están de acuerdo con la posición de sus pares: "Manifestamos que la firma en que el titular de proyecto (...) presenta que miembros de nuestra comunidad habrían llegado a acuerdo sobre las medidas mencionadas previamente. En este sentido manifestamos que la opinión de dichos socios no refleja la opinión de la comunidad, sino que es su opinión individual" (SEA, 2018).

La consulta indígena evidencia, por lo tanto, las fracturas dentro del grupo indígena afectado que terminan por dividir a la comunidad: por un lado, los que defienden el proyecto, y que han realizado negociaciones previas con el titular del proyecto y, por el otro, los que rechazan la propuesta y que buscan negociar la no realización del proyecto mediante la consulta indígena. Además, estos últimos rechazan la negociación individual ejecutada con la empresa como violación a las estructuras de gobernanza de su comunidad. Se construyen, por lo tanto, in-y outgroups dentro de la misma comunidad indígena (Höhl, 2017), que movilizan diferentes relaciones hombre-naturaleza en el marco de la construcción de sus identidades étnicas. Por lo tanto, las posiciones indígenas no son claramente definidas ni homogéneas.

En este contexto, acorde a Wilson (2014), es necesario considerar las relaciones de poder desiguales que privilegian ciertas relaciones socioculturales, ya que se puede argumentar que diferentes grupos usan elementos similares en torno a visiones y prácticas acerca del medio ambiente, pero con un interés estratégico diferente. Los pueblos indígenas pueden usar argumentos metafísicos como elementos de su construcción de identidades y, al mismo tiempo, como armas para atacar políticas hegemónicas de desarrollo y visiones predominantes de la naturaleza (Boelens, 2014). Es así como algunos grupos indígenas reinventan prácticas tradicionales de gobernanza que consideran su conocimiento ecológico heredado para poder oponerse a las percepciones hegemónicas y los proyectos asociados (Latta \& Wittman, 2010). Sin embargo, esa forma de posicionarse no aplica de forma homogénea.

Tal como señala Höhl (2018), las estrategias de los pueblos indígenas son diversas y antagónicas, lo que aumenta la complejidad de las disputas entre los diferentes actores, desafiando los procesos y estructuras de gobernanza existentes. Los diferentes resultados de la consulta indígena demuestran esa complejidad. Mientras que las comunidades Juanita Curipichun de Caren y Camilo Coñoequir, al igual que el Comité de Pequeños Agricultores Pichitrancura y el Comité de Salud Punowemanke Trancura, quienes negociaron previamente con el titular del proyecto, acordaron individualmente recompensaciones que alcanzan un total de 13 millones de pesos chilenos, así como un pacto que compromete un aporte a un fondo de asociatividad de un $1,5 \%$ de las ventas netas anuales que percibe el proyecto (Martínez, 2016), los que se opusieron al proyecto, esperando que la consulta indígena les permitiera impedir la ejecución del proyecto no llegan a ningún acuerdo. Es por esto, por lo que estos últimos presentan un recurso de protección, lo que lleva a la judicialización del proyecto, dado que su postura no tiene implicancias en el proceso final de aprobación del proyecto, debido a que la consulta indígena no tiene carácter vinculante. Por lo consiguiente, el proceso de consulta indígena favorece a los grupos a favor del proyecto, mientras que desfavorece a los que se oponen.

Esto profundiza, por un lado, las divisiones dentro de los grupos indígenas afectados y permite, por el otro, usar las posiciones heterogéneas a favor de la postura hegemónica. Por ende, la fragmentación de la consulta indígena, al realizarse procesos individuales con cada comunidad, pero también al no considerar a todos los actores involucrados en el proyecto en un solo proceso, presenta una limitación en la aplicación del mecanismo, al igual que la falta de un mayor compromiso de los acuerdos alcanzados o no alcanzados. Son estas falencias que contribuyen también a la judicialización en el caso de estudio, lo que causa 
una situación insatisfactoria especialmente para el titular del proyecto, quien, aunque teniendo la aprobación, ve atrasado su calendario y, por ende, se ve confrontado con posibles costos adicionales. A raíz de esta incertidumbre, las empresas mineras, forestales y del sector energético fomentan los procesos previos de negociación con los pueblos indígenas.

\section{Desafíos de gobernanza en la consulta indigena}

Pero, no es solo la judicialización que causa la emergencia de nuevos formatos de negociación entre los diferentes actores. La consulta indígena, al contrario de lo planteado por Kooiman (1999), no involucra necesariamente el Estado, el sector privado y la sociedad civil en el proceso de toma de decisión sobre el control y el uso de los recursos naturales, sino excluye el titular del proyecto, salvo que la metodología que se define entre la comunidad indígena y el SEA lo establezca de otra manera. Esto provoca incertidumbre sobre todo para las empresas del sector privado que las lleva a procesos de participación previos con los grupos indígenas dispuestos. Sin embargo, la falta de incorporación del sector privado en la consulta indígena no solo se debe a la forma de implementación y regulación por parte del Estado chileno, sino también a las recomendaciones de la Organización Internacional de Trabajo (OIT) en el marco del C169 (OIT, 2016). Por lo tanto, se establecen estructuras de gobernanza a nivel global que se contradicen con las realidades locales.

Los encuentros entre los pueblos indígenas y las empresas para negociar directamente acuerdos en relación al proyecto propuesto no se realizan solo una vez presentado el proyecto a la EIA, sino que también previamente e incluso durante la fase de estudio. Aunque la incertidumbre que genera la consulta indígena al excluirlos eventualmente del proceso explica una negociación previa por parte del titular del proyecto, estos procesos son informales pues generan las reglas sobre la marcha. Por lo tanto, se diferencian entre empresas, aunque su objetivo consiste en generar acuerdos formales que se respetan una vez ingresado el proyecto al proceso de EIA y la consulta indígena. Asimismo, se reflejan en la $\mathrm{RCA}^{4}$, tal como es el caso del proyecto hidroeléctrico analizado. La ejecución de un proceso consultivo en la fase del delineamiento del proyecto puede tener la ventaja de que actores locales participen en una modificación del diseño, lo que podría, por ejemplo, evitar futuras judicializaciones. El proceso de EIA, en cambio, no contempla la modificación sustantiva del proyecto ingresado, por ejemplo a raíz de propuestas de los pueblos indígenas radicadas en su relación con la naturaleza, sino que solo tiene como objetivo formular observaciones respecto a la adaptación, mitigación y reparación de impactos ambientales que deben ser consideradas y evaluadas en el informe consolidado de evaluación, con el fin de que el Comité de ministros cuente con la información adecuada para votar sobre la aprobación o el rechazo del proyecto (Leppe, 2015).

En el caso de que las observaciones no son debidamente consideradas, el proceso habilita a los que presentaron observaciones la opción de recurrir a los Tribunales Ambientales competentes para reclamar esa falencia (Leppe, 2015). Por lo tanto, la falta de incidir en el diseño de un proyecto no es solo una falencia de la consulta indígena, sino que del proceso de EIA en general.

Tanto los procesos de negociación por parte de las empresas como la consulta indígena tienen sus propias "reglas del juego". En los procesos de gobernanza realizados previamente por parte de los titulares de los proyectos, estos definen con quién negociar, mientras que los procesos de consulta indígena se aplican para todos los grupos indígenas identificados como afectados por parte de la CONADI, es decir, también aquellos que potencialmente son excluidos de la negociación previa por oponerse al proyecto. Sin embargo, en el proceso de consulta indígena realizado por el SEA, grupos indígenas no registrados por la CONADI pueden seguir excluidos o, por el contrario, pueden haber sido incluidos por las empresas, complejizando el escenario. Tal como vemos en el caso de estudio, la no participación de comunidades indígenas en procesos previos de negociación puede llevar a la judicialización del proyecto y conflictos en los territorios.

Otra falencia que se puede presentar en ambos procesos de negociación identificados corresponde a la presencia de asimetrías de información. Mientras que, en el caso de la negociación directa entre la empresa y las comunidades, ambos actores intercambian información directamente, en el caso de la consulta indígena la información pasa por el SEA como mediador. Ambas situaciones pueden resultar en la retención o pérdida de datos relevantes. Por ejemplo, la falta de conocimiento o bien de comunicación de información por parte del representante del SEA, quien presenta el proyecto a los pueblos indígenas sobre la inclusión del titular del proyecto puede tener consecuencias para el desarrollo de la consulta. Aunque los pueblos indígenas pueden exigir la participación de la empresa, también deben saber que deben precisar su presencia

\footnotetext{
${ }^{4}$ Ver: Resolución Exenta No 177/2015.
} 
explícitamente en la formulación de la metodología de la consulta. Asimismo, la presentación del proyecto por parte del SEA también puede causar asimetrías de información, principalmente debido a la falta de tiempo o la omisión de información relevante o una presentación poco entendible, lo que puede resultar en vacíos de información o problemas de comprensión para la articulación de la posición del grupo indígena (Torres, 2013). Esto rige por ejemplo para la contratación de asesores jurídicos o sociales que debe financiar el SEA si las comunidades así lo solicitan. Sin embargo, para que puedan acceder a ese apoyo deben tener conocimiento sobre esa opción.

En el caso de Añihuerraqui la comunidad Camilo Coñoequir Lloftunecul hizo uso de esta herramienta en el marco de la consulta indígena para fortalecer sus argumentos en contra del proyecto. El apoyo de un antropólogo permitió una (re)significación de sus identidades étnicas y sus relaciones hombre-naturaleza aunque no influyó en la aprobación del proyecto (Silva, 2016).

Al mismo tiempo, al interactuar con el Estado, los pueblos indígenas usan la consulta indígena para articular sus demandas vinculadas a la autodeterminación y la autonomía mediante la articulación de sus relaciones hombrenaturaleza. Por lo tanto, el Estado se ve confrontado a una alta complejidad, ya que los pueblos indígenas presentan demandas en el marco de la consulta indígena para los cuales no fue diseñada y los cuales el SEA no puede responder. Tal como lo demuestra el análisis del proyecto Añihuerraqui, la consulta indígena permite la articulación de una posición frente al proyecto. Sin embargo, la consulta no es de carácter vinculante, es decir, en caso de rechazar el proyecto esto se contempla como una opinión que puede, pero no debe ser tomada en cuenta. Concluyo, por lo tanto, que la consulta indígena visibiliza a los pueblos indígenas en los procesos de toma de decisión. Sin embargo, el objetivo de buscar acuerdos fortalece la posición de aquellos a favor del proyecto frente a los que lo rechazan. Asimismo, las negociaciones previas empoderan los pueblos indígenas, sin embargo, dejan afuera los opositores al proyecto en el caso de que ellos o la empresa decida darle fin a las negociaciones.

\section{Conclusiones}

El análisis del proceso de la consulta indígena, mediante la revisión cualitativa de documentos emitidos en el marco de la evaluación del proyecto hidroeléctrico Añihuerraquí, demuestra que el mecanismo de gobernanza no considera la heterogeneidad que caracteriza a los pueblos indígenas, lo que ha sido un punto de crítica en los últimos años
(Coombes et al., 2013; Von der Porten et al., 2015). En el caso del proyecto analizado, esto provoca que los que están a favor de la propuesta y que son identificados como potenciales contrapartes en el proceso de negociación, sí son empoderados mediante la consulta indígena. Sin embargo, la opinión de los opositores no es considerada en el proceso de toma de decisión final. En consecuencia, se quiebra el tejido social dentro de y entre las comunidades, generando frustración y pérdida de confianza, lo que finalmente deslegitimiza la consulta indígena.

Asimismo, el análisis demuestra que el rol de los pueblos indígenas en los procesos de negociación sobre los recursos naturales en sus territorios no es modificado, ya que se mantiene la subordinación a los intereses hegemónicos, al no incorporar obligatoriamente sus visiones y posturas en la toma de decisión final. Para profundizar en este aspecto, la información obtenida mediante la revisión de los documentos podría ser complementada a futuro por entrevistas, lo que podría contribuir a un mayor entendimiento de cómo las estructuras de gobernanza que sostienen los tejidos sociales son modificadas mediante el mecanismo, provocando, por ejemplo, quiebres de relaciones.

Existen, además, otros factores importantes que contribuyen a la deslegitimación de la consulta indígena: la participación indígena se limita a la consulta y no tiene implicancias para la votación final del proyecto. Lo anterior condiciona el mecanismo al no ofrecer la posibilidad de proponer modificaciones o alternativas al proyecto que lo podrían hacer viable y compatible con las socionaturalezas asociadas a las identidades étnicas de los pueblos indígenas afectados $\mathrm{y}$, por lo tanto, contribuir a un mayor control de los recursos naturales en territorios indígenas. Esto, junto con la falta de espacios para la articulación de otras demandas fuera del contexto del proyecto, provoca que los pueblos indígenas usen el espacio y la oposición al proyecto como vehículo para sus intereses centrados en la autodeterminación y autonomía en sus territorios. Por lo tanto, la consulta indígena acelera procesos de redefinición y reetnificación, específicamente en territorios que no habían sido confrontados con proyectos extractivistas, visibilizando sus posturas, pero sin necesariamente empoderarlas. Esto último está vinculado a la alta fragmentación del mecanismo.

Las diversas negociaciones paralelas, en lugar de aprovechar las estructuras de gobernanza existentes dentro de los territorios indígenas y juntar sus representantes en un proceso de negociación más amplio e inclusivo, debilitan la consulta y la deslegitimizan. Posiblemente, otras transformaciones de los procesos y estructuras de gobernanza ambiental, tales como un rediseño de la consulta, pero también del 
proceso de EIA haciendo la participación indígena pero también ciudadana, más inclusiva y vinculante, podría lograr una menor judicialización de los proyectos de inversión en el futuro. Sin embargo, estos cambios implican que el poder hegemónico que fomenta un desarrollo basado en el crecimiento acceda a un mayor involucramiento de otros actores en igualdad de condiciones en los procesos de toma de decisión, lo que permite llegar a consensos respecto a la (no)implementación de proyectos hidroeléctricos en territorios indígena generando acuerdos en lugar de provocar conflictos basados en desequilibrios de poder, asimetrías de información y conocimiento.

\section{Referencias}

Aliste, E., Folchi, M. \& Núñez, A. (2018). Discourses of Nature in New Perceptions of the Natural Landscape in Southern Chile. Frontiers in Psychology, 9, 1-16. https://doi.org/10.3389/fpsyg.2018.01177

Armitage, D. (2008). Governance and the commons in a multi-level world. International Journal of the Commons, 2(1), 7-32. http://doi.org/10.18352/ijc.28

Barría, J. (2019). La consulta indígena en la institucionalidad ambiental de Chile: Consecuencias para la minería y las comunidades indígenas Collas de la Región de Atacama. Investigaciones Geográficas, 57, 76-93. https://doi.org/10.5354/07195370.2019 .53490

Bebbington, A. (ed.) (2012). Social Conflict, Economic Development and Extractive Industry. Evidence from South America. Oxon: Routledge.

Berkes, F. (2010). Devolution of environment and resources governance: trends and future. Environmental Conservation, 37(4), 489-500. https:// doi.org/10.1017/S037689291000072X

Boelens, R. (2014). Cultural politics and the hydrosocial cycle: Water, power and identity in the Andean highlands. Geoforum, 57, 234-247. https://doi. org/10.1016/j.geoforum.2013.02.008

Bridge, G. \& Perreault, T. (2009). Environmental Governance. En Castree, N., Demeritt, D., Liverman, D. \& Rhoads, B. (eds). A Companion to Environmental Geography (pp. 475-497). West Sussex: Blackwell Publishing Ltd.
Castillo, M., Espinoza, C. \& Campos, L. (2017). Régimen de desigualdad y pueblos indígenas en el período postdictatorial. Tres vías en la disputa por la igualdad. Estudios Atacameños, 54, 217-237. Recuperado de https://revistas.ucn.cl/index.php/ estudios-atacamenos/article/view/1719

CONADI (3 de enero de 2013). Oficio $\mathrm{N}^{\circ} 336$. Recuperado de http://seia.sea.gob.cl/ archivos/ CONADI_EIA_Anihuerraqui.pdf

Coombes, B., Johnson, J. \& Howitt, R. (2013). Indigenous geographies II: The aspirational spaces in postcolonial politics - reconciliation, belonging and social provision. Progress in Human Geography, 37 (5), 691-700. https://doi.org/10.1177/0309132512469590

Cronin, A. \& Ostergren, D. (2007). Tribal Watershed Management. Culture, Science, Capacity, and Collaboration. American Indian Quarterly, 31 (1), 87-109. https://doi.org/10.1353/aiq.2007.0004

De Loë, R. \& Patterson, J. (2017). Rethinking Water Governance: Moving Beyond Water-Centric Perspectives in a Connected and Changing World. Natural Resources Journal, 57(1), 75-99. Recuperado de https://digitalrepository.unm.edu/nrj/vol57/iss1/4

Decreto 40, Aprueba reglamento del sistema de evaluacion de impacto ambiental. Santiago Chile, 12 de agosto de 2013. Recuperado de https://www. leychile.cl/Navegar?idNorma=1053563

Donoso, S. (2014). Empresas y comunidades indígenas: el Nuevo escenario que plantea el Convenio 168 de la OIT. Temas de la agenda pública, 73, 1-20. Recuperado de https://politicaspublicas.uc.cl/wpcontent/uploads/2015/02/empresas-y-comunidadesindigenas.pdf

Escobar, A. (2006). Difference and Conflict in the Struggle Over Natural Resources: A political ecology

framework. Development, 49, 6-13. https://doi. org/10.1057/palgrave.development.1100267

Gestión Ambiental Consultores (GAC). (2012). Estudio de Impacto Ambiental. Santiago de Chile: GAC. 
Garrido, C. (14 de Julio de 2015). Por 7 votos contra 4 aprueban construir central hidroeléctrica en Curarrehue. Diario Austral de Teтисо. Recuperado de http://www.soychile.cl/Temuco/ Sociedad/2015/07/14/334111/

Harvey, D, (2007). Breve historia del neoliberalismo. Madrid: Akal.

Höhl, J. (2018). Hidroelectricidad y pueblos indígenas: Un análisis del megaproyecto Ralco en la región Bío Bío, Chile. In Agua y disputas territoriales en Chile y Colombia (pp. 297-333). Bogotá: Serie Perspectivas Ambientales de la Facultad de Ciencias Humanas de la Universidad Nacional de Colombia-Grupo Cultura y Ambiente.

Höhl, J. (2017). ¿Lucha por recursos o lucha por territorio? Conflictos por agua y energía en la Araucanía. In Violencia y desigualdad (pp. 191-208). Buenos Aires: Nueva Sociedad: Friedrich-EbertStiftung: ADLAF.

Höhl, J. (2015). , Wir sind keine Chilenen, wir sind Mapuche". Die Mapuche im Spannungsfeld zwischen Staat, Wirtschaft und Gesellschaft, 1973-1997. Stuttgart: Verlag Hans-Dieter Heinz.

International Labour Organization (ILO). (1989). Indigenous and Tribal Peoples Convention, C169. Recuperado de https://www.ilo.org/dyn/normlex/en/ $\mathrm{f} ? \mathrm{p}=$ NORMLEXPUB:12100:0::NO::P12100_ILO CODE:C169

Kaltmeier, O. (2004). Die Mapuche: Brüche und Einbrüche zwischen Widerstand und Eroberung. En Imbusch, P., Messner, D. \& Nolte, D (eds). Chile heute (pp. 191-206). Frankfurt: Vervuert.

Kooiman, J. (1999). Social-Political Governance. Public Management: An International Journal of Research and Theory, 1(1), 67-92. https://doi. org/10.1080/14719037800000005

Latta, A. \& Wittman, H. (2010). Environment and citizenship in Latin America: A new paradigm for theory and practice. European Review of Latin American and Caribbean Studies, 89, 107-116. Recuperado de https://www.jstor.org/stable/20788578
Leff, E. (2000). Espacio, lugar y tiempo: la reapropiación social de la naturaleza y la construcción local de la racionalidad ambiental. Desenvolvimento e Meio Ambiente, 1, 57-69. https://doi.org/10.5380/dma. v1i0.3057

Leppe, J. (2015). Consulta Indígena y procedimiento de evaluación de impacto ambiental. Análisis de una relación normativa. Revista de Derecho de la Pontificia Universidad Católica de Valparaíso, 44, 369-384. _ _ https://doi.org/10.4067/S071868512015000100011

Levil, R. (2006). Sociedad Mapuche Contemporánea. En Marimán, P., Caniuqueo, S., Millalén, J. \& Levil, R. i...Escucha, winka...! Cuatro ensayos de Historia Nacional Mapuche y un epílogo sobre el futuro (pp. 219-252). Santiago: LOM Ediciones.

Ley sobre Bases Generales del Medio Ambiente. Santiago, Chile, 9 de marzo de 1994. Recuperado de https://www.leychile.cl/Navegar?idNorma=30667

Li, T. (2010). Indigeneity, Capitalism, and the Management ofDispossession. Current Anthropology, 51 (3), 385-414. https://doi.org/10.1086/651942

Lillo, R. \& Nolden, H. W. (2003). ¿Conflicto étnico chileno en las tierras del Cono Sur? Temuco: Universidad Católica de Temuco.

Martínez, C. (2016). Suprema rechaza recurso de protección contra una central hidroeléctrica en Curarrehue. BioBio Chile. Recuperado de http:// www.biobiochile.cl/noticias/2016/01/03/supremarechaza-recurso-de-proteccion-contra-una-centralhidroelectrica-en-curarrehue.shtml

Mette Kjaer, A. (2011). Rhodes' Contribution to Governance Theory: Praise, Cristicism and the future governance debate. Public Administration 89 (1), 101-113. https://doi.org/10.1111/ j.14679299.2011.01903.x

Moore, M. L., von der Porten, S. \& Castleden, H. (2017). Consultation is not consent: hydraulic fracturing and water governance on Indigenous lands in Canada. WIREs Water, 4 (1) https://doi.org/10.1002/wat2.1180 
Organización Internacional del Trabajo. (2016). Reporte Regional. Recuperado de https://www. ilo.org/wcmsp5/ groups/public/---americas/---rolima/documents/publication/wcms_507556.pdf $(18.05 .2020)$

Peluso, N. L. (2012). What's Nature Got to Do With it? A Situated Historical Perspective on Socionatural Commodities. Development and Change, 43(1), 79-104. https://doi.org/10.1111/j.14677660.2012.01755.x

Peralta, P., Bebbington, A., Hollenstein, P., Nussbaum, I. \& Ramírez, E. (2015). Extraterritorial Investments, Environmental Crisis, and Collective Action in Latin America. World Development, 73, 32-43. https://doi. org/10.1016/j.worlddev.2014.08.020

Pierre, J. \& Peters, B. G. (2000). Governance, Politics, and the State. London: Macmillan Press Ltd.

Resolución Exenta No 177/2015. Recuperado de http://seia.sea.gob.cl/archivos/2015/07/22/CH_ Anihuerraqui _VF.pdf (18.05.2020).

Rhodes, R.A.W.(1996). TheNew Governance: Governing without Government. Political Studies, 44(4), 652667. https://doi.org/10.1111/j.1467-9248.1996. tb01747.x

Rivera, C. (2011). Conflictos ambientales y redes transnacionales de defensa en el Chile Postdictadura. Revista de Ciencias Sociales, 17(2), 231246. Recuperado de https://www.redalyc.org/ pdf/280/28022757004.pdf

Rojas, A., Sabatini, F. \& Sepúlveda, C. (2003). Conflictos ambientales en Chile: aprendizajes y desafíos. Revista Ambiente y Desarrollo del CIPMA, 19(2), 22-30. Recuperado de http://biblioteca.cehum. org/handle/CEHUM2018/1354

SEA. (2018). Servicio de Evaluación Ambiental. Recuperado de http://sea.gob.cl/

Silva, H. (Cord.) (2016). Los impactos en derechos humanos de los proyectos de inversión hidroeléctrica en el territorio mapuche de la Región de la Araucanía. Temuco: Observatorio de Derechos de los Pueblos Indígenas.
Swyngedouw, E. \& Copano, M. Z. (2018). Politizando las ecologías políticas urbanas. Investigaciones Geográficas, 56, 153-167. https://doi. org/10.5354/0719-5370.2018.51996

Swyngedouw, E. (2011). Whose environment? The end of nature, climate change and the process of postpolitization. Ambiente \& Sociedade, 14(2), 69-87. https://doi.org/10.1590/S1414-753X2011000200006

Swyngedouw, E. (2004). Scaled geographies: Nature, place, and the politics of scale. Scale and geographic inquiry: Nature, society, and method, 129-153. https://doi.org/10.1002/9780470999141.ch7

Torres, V. (2013). Acceso a la información, participación y justicia en temas ambientales en América Latina y el Caribe. Situación actual, perspectivas y ejemplos de buenas prácticas, serie Medio Ambiente y Desarrollo. CEPAL: Santiago de Chile.

Treib, O., Bähr, H. \& Falkner, G. (2007). Modes of governance: towards a conceptual clarification. Journal of European Public Policy 14 (1), 1-20. https://doi.org/10.1080/135017606061071406

Ulloa, A. (2020). The rights of the Wayúu people and water in the context of mining in La Guajira, Colombia: demands of relational water justice. Human Geography 13(1), 6-15. https://doi. org/10.1177/1942778620910894

Von der Porten, S., de Loë, R. C. \& Plummer, R. (2015). Collaborative Environmental Governance and Indigenous People: Recommendations for Practice. Environmental Practice, 17(2), 134-144. https://doi. org/10.1017/S146604661500006X

Walter, M. \& Urkidi, L. (2011). Dimensions of environmental justice in anti-gold mining movements in Latin America. Geoforum, 42(6), 683-695. https:// doi.org/10.1016/j.geoforum.2011.06.003

Wilson, N. J. (2014). Indigenous water governance: Insights from the hydrosocial relations of the Koyukon Athabascan village of Ruby, Alaska. Geoforum, 57, 1-11. https://doi.org/10.1016/j. geoforum.2014.08.005

Yin, R.K. (2009). Case Study Research. Design and Methods $\left(4^{\text {th }}\right.$ ed.). Thousand Oaks: Sage Publications. 


\section{Agradecimientos}

Agradezco el apoyo de José Miguel Valdés con las cartografías y los aportes por parte de Jessica Barría en torno a ideas centrales del artículo. Asimismo, quisiera agradecer los comentarios recibidos por parte de Michael Handke y Michael Lukas, y los revisores externos a versiones anteriores de este artículo. 